Deutsche Zeitschrift f. Nervenheilkunde, Bd. 168, S. 183-194 (1952).

Aus dem neuropathologischen Laboratorium der psychiatrischen Klinik

(Direktor: Prof. Dr. R. W. Waggoner)

und der neurologischen Klinik der Universität Michigan in Ann Arbor, Michigan/USA

(Direktor: Prof. Dr. R. DE JoNG)

\title{
Haliervordey-Spatzsche Krankheit*.
}

Von

\section{K. Scharenberg und R. De Jong.}

Mit 8 Textabbildungen.

(Eingegangen am 10. März 1952.)

Das von Hallervorden u. Spatz im Jahre 1922 eingehend beschriebene Krankheitsbild ist im Laufe von 30 Jahren nur selten beobachtet worden. Die Krankheit trat mehrfach familiär auf (HALLERvordesSpatz, Kalinowski, Winkelmax). Auf der anderen Seite sind aber auch sporadische Fälle beobachtet worden, die klinisch und anatomisch mit den Beobachtungen von Hallervorden u. Spatz weitgehend übereinstimmen. Wir sind in der Lage, dieser letzteren Gruppe einen weiteren Fall hinzuzufügen, was bei der Seltenheit diesbezüglicher Beobachtungen berechtigt erscheint.

$J$. S. G., ein 14 jähriger Knabe wurde als zweites Kind eines Hochschullehrers geboren. Ein 4 Jahre älterer Bruder, eine 3 Jahre jüngere Schwester und die Eltern sind gesund. Nerven- und Geisteskrankheiten sind in der Familie angeblich nicht vorgekommen. Schwangerschiaft, Geburt und die ersten Lebensmonate des Pat. wurden von den Eltern, bis auf eine kurzdauernde postnatale Cyanose, als normal geschildert. Der Junge saß 3 mit 6 Monaten, konnte im Alter von 14 Monaten laufen und mit 2 Jahren sprechen. Körperlich soll er jedoch immer unterentwickelt gewesen sein. Das Laufen sei ihm sichtlich schwer gefallen und auch die Sprache wäre nie ganz deutlich gewesen. Im Alter von 3 Jahren litt das Kind an Mumps, mit 31/2 Jahren traten Varizellen auf, mit 4 Jahren hatte es eine Claviculafraktur und mit 41/2 Jahren wurden ihm die Tonsillen entfernt. Nach Aussagen der Eltern zu urteilen, soll das Kind bereits mit 3 Jahren gestottert haben; zur selben Zeit wurde der Gang auffallend ungeschickt, die ganze Motorik unbeholfen. Im Laufe der nächsten Jahre wurde das Gehen immer beschwerlicher, das Kind mußte sich an Wänden und Möbeln festhalten, stürzte trotzdem oft zu Boden und fiel dabei meist nach rechts. Mit 5 Jahren litt der Junge an einer fieberhaften Erkrankung mit schwerem Erbrechen, die als Acidose gedeutet wurde.

Bei der ersten neurologischen Untersuchung im Alter von 8 Jahren wurden folgende Befunde erhoben: Unterernährter, schwächlicher Junge mit auffallend großem Kopf, der ohne Unterstützung nicht gehen konnte. Bei Prüfung des Bewegungsablaufes wurden die Arme hilflos hin- und hergeworfen, es bestand eine schwere allgemeine Koordinationsstörung. Der Finger-Nase-Versuch konnte auch mit offenen Augen nicht richtig durchgeführt werden. Zeitweilig traten spontane choreoathetotische Bewegungen der Hände auf. Bei deutlich herabgesetztem Muskel-

* Herm Prof. Dr. J. Hallenvorden zum 70. Geburtstag gewidmet. 
dehnungswiderstand waren alle Sehnenreflexe lebhaft gesteigert. Beiderseits konnten Fuß- und Patellarkloni ausgelöst werden. Das Hofrmannsche Zeichen war positiv. Bauchhaut- und Cremasterreflexe fehlten. Die Sensibilität war völlig in Ordnung. Am Augenhintergrund war kein krankhafter Befund zu erheben. Die Sprache war ataktisch gestört. Der Kranke machte einen geistig stumpfen Eindruck. Er mußte kurz vorher aus der Schule genommen werden. Der Intelligenzquotjent nach STANFORD-BINET betrug 71 .

Untersuchungen des Blutes, Liquors und Urins ergaben keine krankhaften Veränderungen. Röntgenaufnahmen des Schädels zeigten eine verstärkte Zeichung der Diploe. Im Luftencephalogramm stellte sich ein median stehendes, seitengleich erweitertes Ventrikelsystem dar. Elektroencephalographisch fand sich bei einer highvoltage über $100 \mu$-Volt eine durchschnittliche Frequenz von 3 bis $4 \mathrm{hz}$, die gelegentlich auf $2 \mathrm{hz}$ absank. Zeitweilig wurde dieser langsame Rhythmus von 15 bis $18 \mathrm{hz}$-Salven mit steilem Anstieg und regelmäßigen Wellen von niedriger bis mittlerer Spannung unterbrochen oder überlagert.

Diese Befunde wurden als Zeichen einer allgemeinen Hirnschädigung aufgefaßt, eine definitive klinische Diagnose konnte jedoch nicht gestellt werden.

Spätere Nachuntersuchungenim 10. und 11. Lebensjahre zeigten eine stete Verschlechterung des Zustandes. Die Eltern gaben hingegen an, daß der Junge zeitweise besser gehen konnte und sogar in der Lage war, gelegentlich mit seinen Altersgenossen zu spielen. Aber der geistige Verfall wurde immer deutlicher, die Sprache war fast unverständlich; er war nicht mehr in der Lage zu lesen und saß meist still und teilnahmslos im Sessel. Bei jedem Gehversuch kam er zu Fall, wobei er sich häufig verletzte. Die choreoathetotischen Bewegungsstörungen wurden deutlicher. An den Extremitäten war eine spastische Versteifung feststellbar. Seit dieser Zeit traten in zunehmender Häufigkeit Anfälle auf, die zuerst als „petits maux“, später als generalisierte Krampfanfälle imponierten. Das letzte Lebensjahr war durch eine tiefgreifende Verblödung und häufige generalisierte Anfälle mit Urininkontinenz gekennzeichnet. Der Tod trat im Alter von 14 Jahren ein.

Die Allgemeinsektion ergab keinen bemerkenswerten Befund.

Schädel- und Gehirnsektion: Die Schädelkalotte war mit 1,5 cm stark verdickt. Die Knochen der Schädelbasis erschienen massiv und ihre Oberfläche war wellig. Das Gehirn wog in unfixiertem Zustand $790 \mathrm{~g}$. Die weichen Häute waren verdickt und milchig getrübt. Groß- und Kleinhirnwindungen waren verschmälert; die Sulci klafften besonders über dem Stirnhirn auffallend (Abb. 1). Die Brücke war ebenfalls von der allgemeinen Atrophie betroffen, die Medulla oblongata hingegen erschien unverändert. Auf Frontalabschnitten erwiesen sich Mark und Rinde von Groß- und Kleinhirn als stark atrophisch. Die Seitenventrikel waren mäßig erweitert. Globus pallidus und die „,Rote Zone“ der Substantia nigra zeigten eine auffallende braun-grünliche Verfärbung. Im übrigen boten die Stammganglien keine Besonderheiten. Die Oliven erschienen auffallend weiß und waren von der Umgebung schlecht abzugrenzen. 
Histologische Untersuchung: Im NIssL-Bild ist eine schwere Störung der Rindenarchitektonik des Großhirns zu erkennen. Die Ganglienzellen

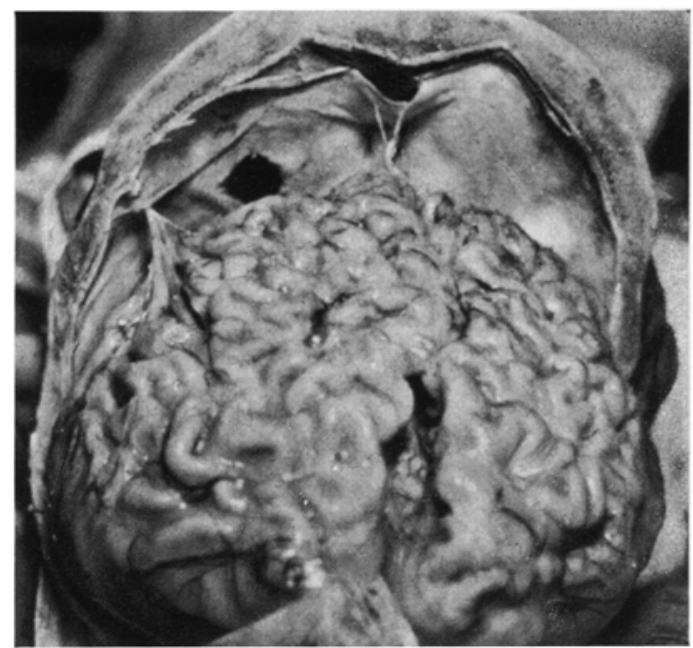

Abb. 1. Gehirn in situ, schlaff und atrophisch. Calotte stark verdickt.

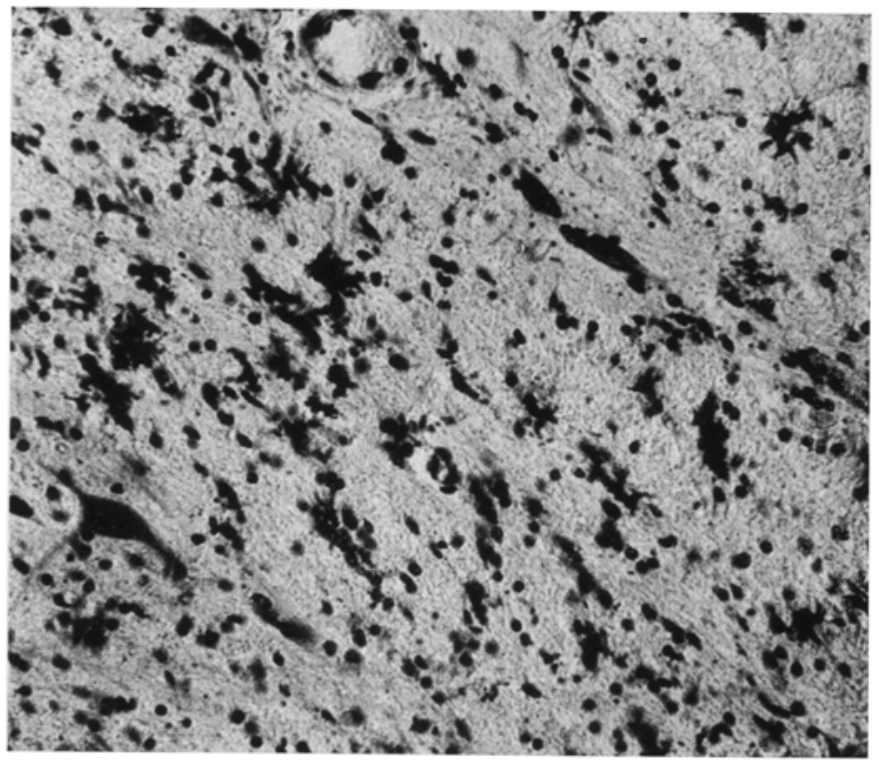

Abb. 2. NIssL-Färbung. Substantia nigra mit Pigmentherden.

sind besonders in der dritten und vierten Schicht ausgefallen. Die Purkinjezellen sind ebenfalls erheblich rarifiziert. Körnerzellschicht und 
Dentatumband sind stark gelichtet. Die Marklager sind durch ihren Kernreichtum gekennzeichnet. Im Pallidum und in der roten Zone des Substantia nigra finden sich große Mengen eines grünlich-schwarzen Pigments (Abb. 2), das meist von Gliazellen aufgenommen worden ist. Das Parenchym dieser Formationen ist ganz erheblich vermindert, es finden sich nur noch vereinzelte Ganglienzellen. Weniger stark ist der

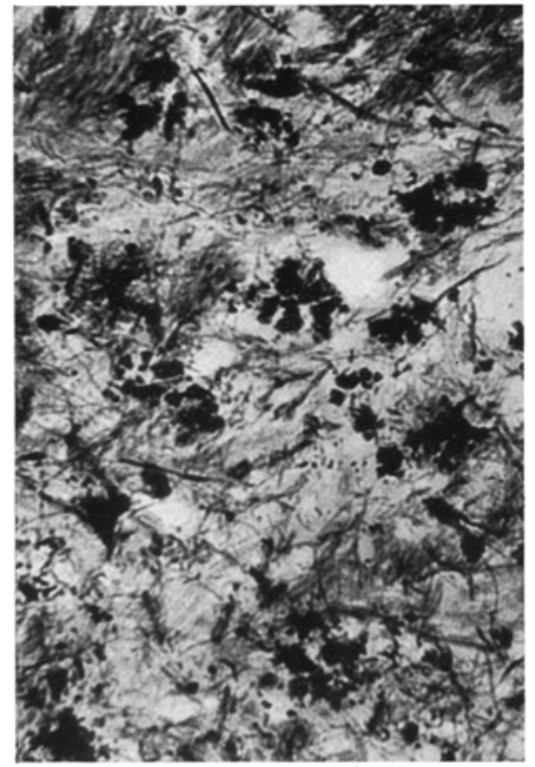

a

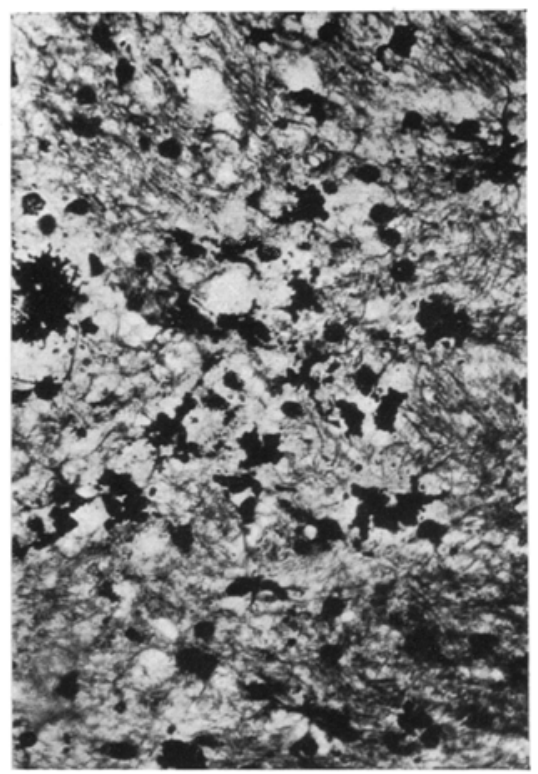

b

Abb. 3a. Silberkarbonat, nicht reduzierte Variante. Pallidum mit zahlreichen Pigmentherden und diffus gefärbtem, degeneriertem Mark.

Abb. 3b. Silberkarbonat, Astrogliatechnik. Pallidum mit Gliafilz und Pigmentherden.

Parenchymverlust im Striatum und in den übrigen Stammganglienbezirken. Ein kleiner Pigmentherd findet sich noch in den oberen Teilen des Claustrums. In den Stammganglien, besonders ausgeprägt in den Gebieten der Pigmentherde, ist die Glia vermehrt. Häufig lassen sich proliferative Formen erkennen. Bemerkenswert erscheint ferner, da $\boldsymbol{B}$ sich in der kompakten Zone der Substatia nigra nur wenig melanotisches Pigment nachweisen läßt.

Die weichen Hirnhäute zeigen eine zellarme Fibrose. Die Gefäße erscheinen normal, nur im Stammganglienbereich finden sich einzelne wenig ausgeprägte perivaskuläre Rundzellinfiltrate, zweifellos das anatomische Substrat einer ,,symptomatischen Entzündung“.

Mit Hilfe der Silberkarbonattechnik war es möglich, diese Befunde weiter zu verfolgen. So zeigt die, nicht reduzierte Variante“ im Pallidum 
nur noch wenige erhaltene Markfasern, kenntlich an der schwarzen Imprägnation der Achsenzylinder. Die zerstörten Myelinstrukturen sind dagegen nur zartrosa imprägniert und bilden einen homogenen Untergrund. In das Netzwerk der verbliebenen Markfasern sind die Pigmentherde, die aus verschieden großen Pigmentkugeln bestehen, und oftmals ein recht bizarres Aussehen haben. unregelmäßig eingelagert (Abb. 3a).

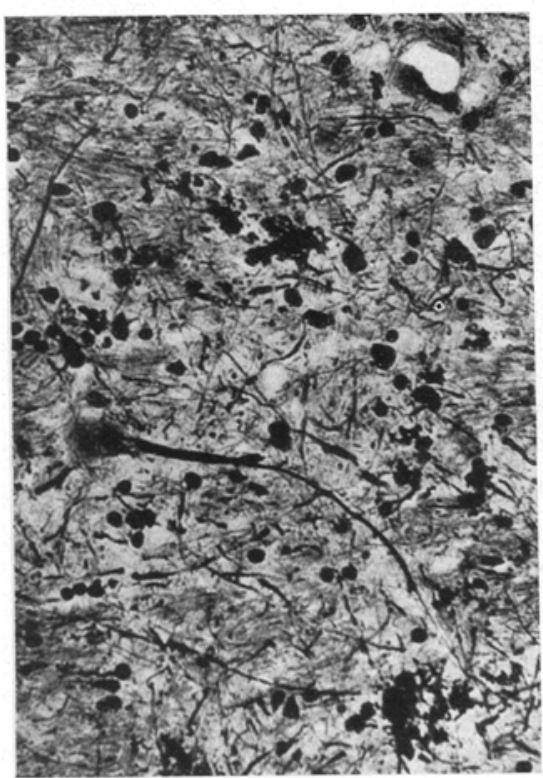

a

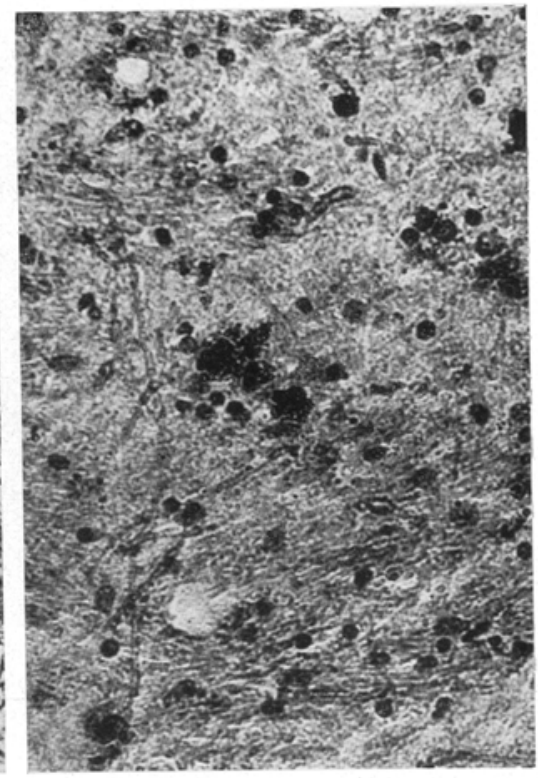

b

Abb. 4a. Silberkarbonat, 3fache Variante. Pallidum. In der Uningebung der erhaltenen Ganglienzelle Pigmentherde.

Abb. 4b. Scharlachrotfärbung. Pallidum. Die Pigmentherde enthalten reichlich Neutralfette.

Die ,Astrogliavariante“ läßt einen dichten, gleichmäßigen Faserfilz mit zahlreichen proliferierten, oftmals auch degenerierten Astrocyten erkennen (Abb. 3b). Vielfach sind die Pigmentstoffe von Astrocyten phagocytiert worden, was das teilweise bizarre Aussehen der Pigmentherdchen erklärt. Im fortgeschrittenen Zustand der Pigmentspeicherung scheinen die Astrocyten unter Hinterlassung des in charakteristischer Form gespeicherten Pigments zu zerfallen.

Mit der ,dreifachen Imprägnation" zur Darstellung der Neuronen lassen sich nur noch einzelne geschrumpfte Ganglienzellen im Pallidum nachweisen (Abb. 4a). Ein histologisch identisches Bild besteht auch in der roten Zone der Substantia nigra, doch ist hier der Markzerfall nicht so ausgesprochen. 
Bei der Eisenreaktion mit Berlinerblau erscheinen Pallidum und Substantia nigra schon makroskopisch blau gefärbt. Histologisch ist das Eisen sowohl in den Pigmentherden, als auch diffus im Gewebe nachweisbar. Scharlachrotfärbungen demonstrieren große Mengen von Neutralfetten in den befallenen Gebieten (Abb. 4b).

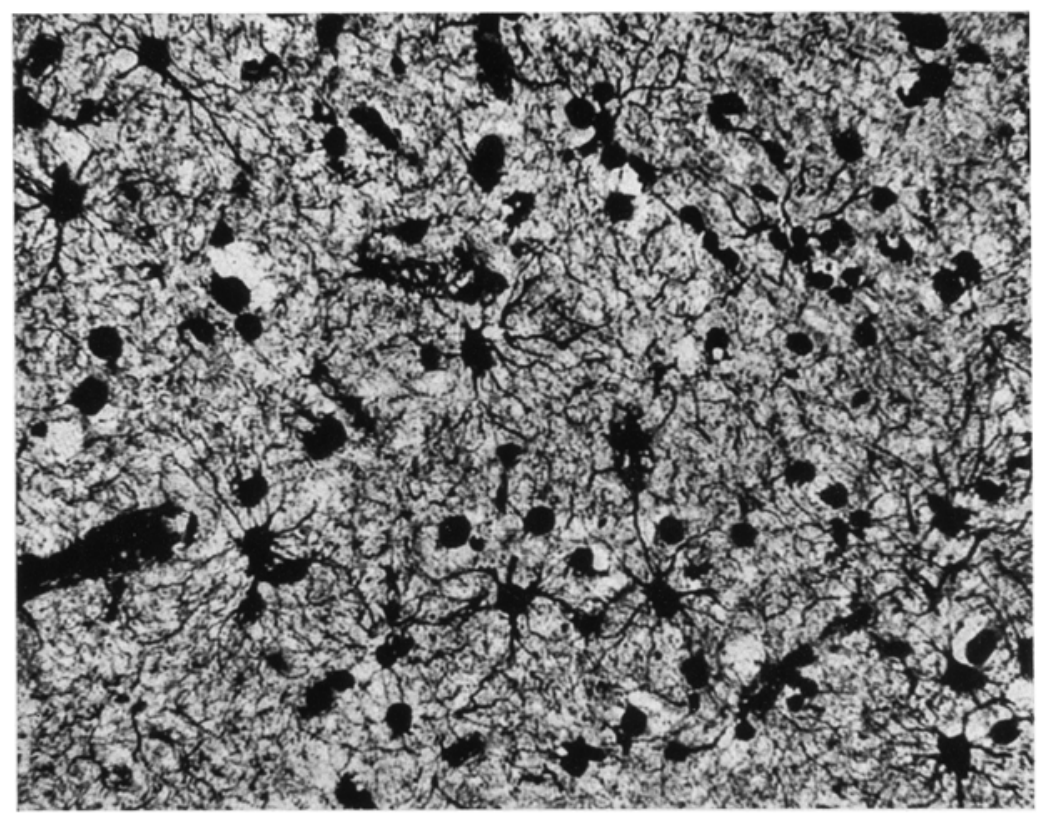

Abb. 5. Astrogliatechnik. Astrogliafilz im Putamen.

Corpus striatum und Thalamus sind von einem dichten astrocytären Faserfilz durchdrungen (Abb. 5). Auch in diesen Regionen sind die Ganglienzellen ausgefallen oder geschrumpft.

Die Großhirnrinde läßt eine starke Lichtung der Ganglienzellen bei Anwendung der dreifachen Imprägnation erkennen. In der zweiten Schicht besteht teilweise ein status spongiosus, die dritte und vierte Schicht zeigen eine besonders ausgesprochene Ganglienzellverödung (Abb. 6).

Im Mark ist die Glia sehr aktiv. Úberall findet man große Mengen proliferierter, teilweise auch degenerierter Astro-, Mikro- und Oligodendrogliazellen (Abb. 7). Auch das Mark ist erheblich gelichtet.

Das Kleinhirn ist vom Prozeß auch schwer betroffen. Das histologische Bild ist so kompliziert, daß es hier nur in Grundzügen geschildert werden kann. Die Purkinjezellen sind in vielen Windungen bis auf einige wenige Exemplare ausgefallen. Das Netzwerk ihrer Dendriten in der Molekularschicht ist gelichtet. Die übriggebliebenen Korbzellen sind geschwollen 
(Abb. 8). Bemerkenswert ist der Untergang der Korbgeflechte, welche entweder völlig verschwunden oder bis auf wenige Fasern reduziert sind, so daß die Purkinjezellen auch da, wo sie noch in größerer Anzahl erhalten sind, nicht mehr ihre charakteristischen Faserkörbe erkennen

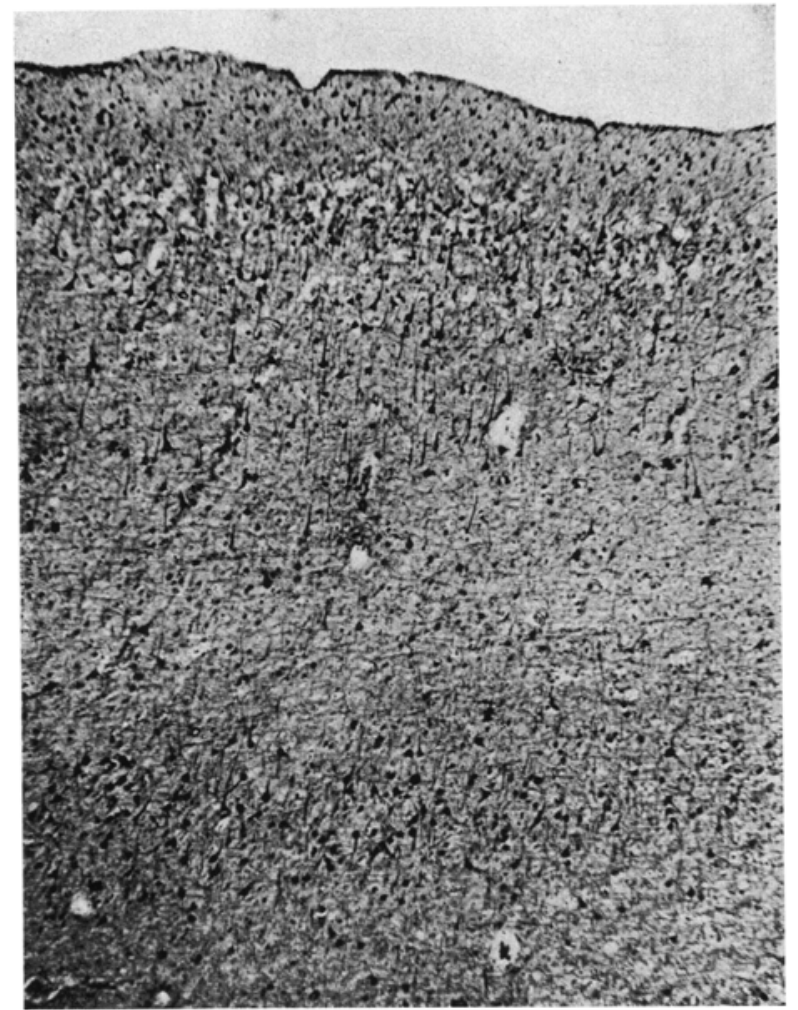

Abb. 6. 3fache Variante. Rinde. Schwere Störungen in der Cytoarchitektonik mit laminären Ausfällen in der dritten und vierten Schicht.

Iassen. Die von den Kollateralen der Purkinjezellneuriten gebildeten Plexus sind kaum mehr darstellbar. Die Körnerzellen sind stark reduziert. Moos- und Kletterfasern bilden ein nur dürftiges Netzwerk, während Glomeruli gar nicht mehr erkennbar sind.

Das Parenchym des Nucleus dentatus ist dem Prozeß großenteils zum Opfer gefallen. Das Dentatumband ist von einer dichten fasrigen Gliose durchsetzt. Auch im Kleinhirnmark läßt sich ein Ausfall an Markfasern und eine Proliferation der Gliazellen nachweisen.

In den Oliven findet sich bei gut erhaltenen Ganglienzellen eine dichte Astrogliose mit starker Faserbildung, ebenso in der Substantia reticularis der Medulla oblongata. 


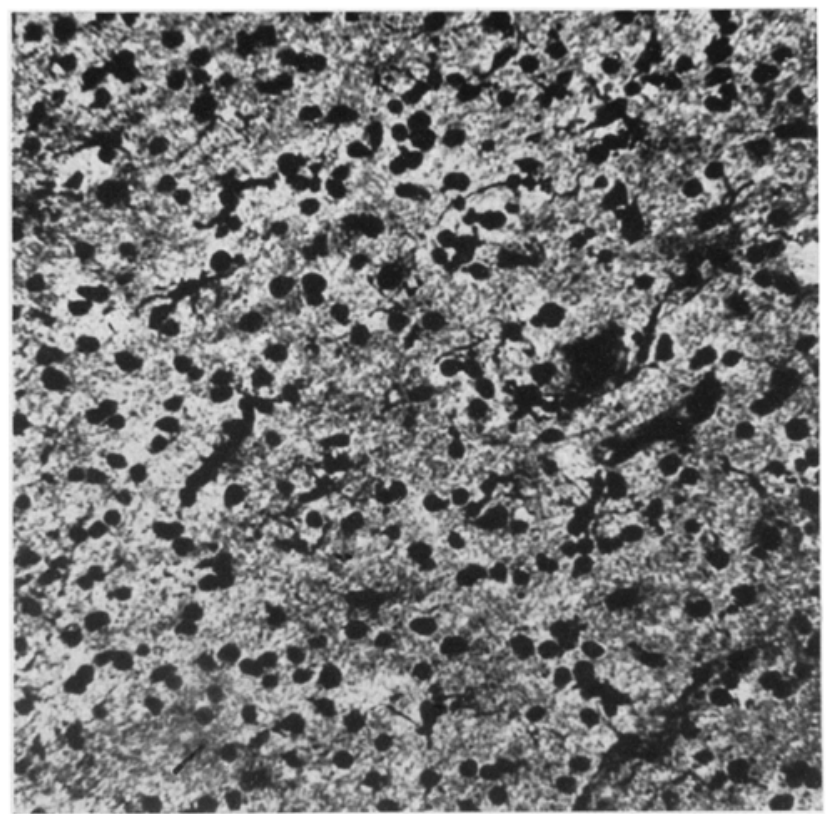

Abb. 7. Astrogliatechnik. Mark. Diffuse Wucherung von Astro-, Mikro- und Oligodendroglia.

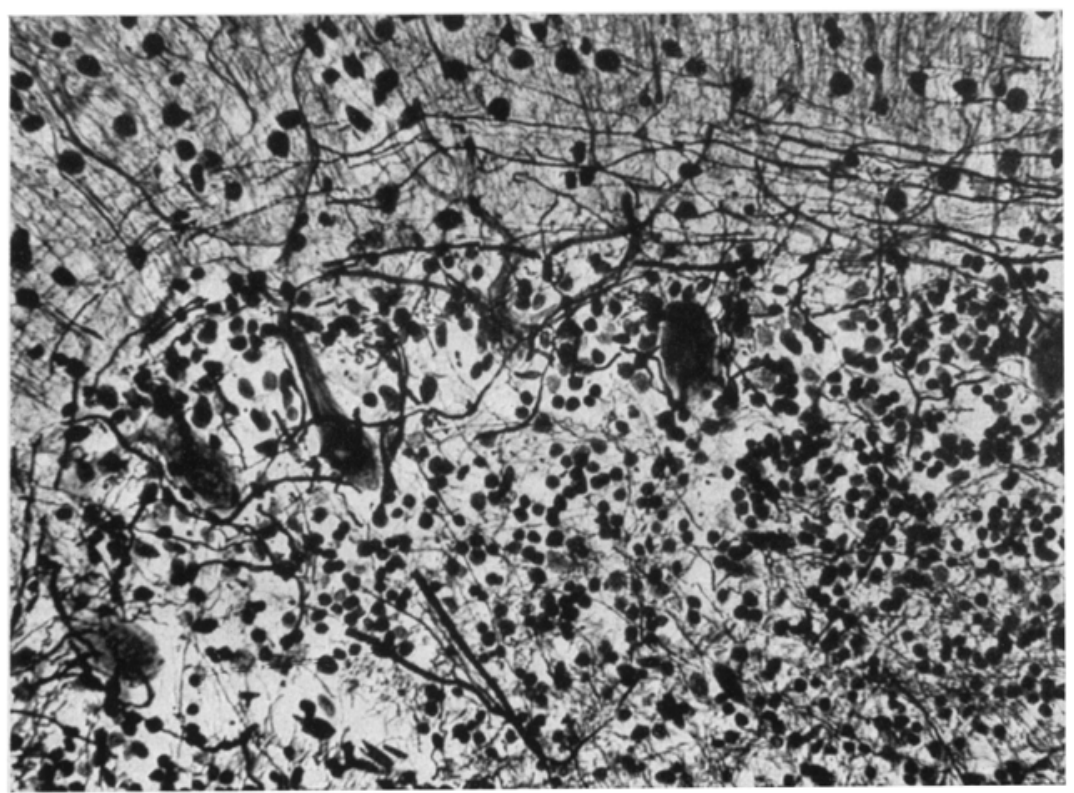

ApD. ช. sracne variante. veredellum mit schweren austallen der vanglienzellen in allen Schichten und Degeneration der Neuriten und Dendriten. 
Das Rückenmark stand der Untersuchung nicht zur Verfügung.

Die hier mitgeteilten klinischen und anatomischen Befunde stimmen mit den bisherigen Beobachtungen von Hallervorden-Spatzscher Krankheit so weitgehend überein, daß wir nicht zögern, unsere Beobachtung diesem Krankheitsbild zuzordnen. Wenn unser Fall auch keinerlei familiäre Belastung zeigte, so möchten wir doch nicht bezweifeln, daß wir es hier mit einem einheitlichen Krankheitsbild zu tun haben. Hierfür spricht neben dem großen Prozentsatz der familiären Beobachtungen vor allem die klinische Einheitlichkeit von Symptomatologie und Prozeßablauf und die in allen Fällen charakteristischen anatomischen Befunde im Gehirn.

In den meisten bekannt gewordenen Fällen trat die Krankheit im ersten Dezennium,, nur selten etwas später, auf, nahm einen chronischprogredienten Verlauf und endete nach 10-20 Jahren mit dem Tod. Ein Alter von 30 Jahren hat nur der von Yakovlev beobachtete Kranke erreicht.

Die Symptomatologie ist gekennzeichnet durch Versteifung der Extremitäten, zunehmende organische Sprachstörung, geistigen Verfall, athetotische Bewegungen und Pyramidenzeichen. Einige weitere neurologischen Ausfallerscheinungen sind nicht mit derselben Konstanz gefunden worden. So schildern Kalinowski, Benda, Netski u.a. Sehstörungen auf Grund einer Opticusatrophie, während in den Fällen von Winkelman eine Retinitis pigmentosa vorgelegen hatte. Klumpoder Hohlfußbildung sind recht häufig beobachtet worden, während Torsionsspasmus (Osman u. Schükrü), Ataxie (Netsky und Mitarbeiter) sowie Augenmuskelstörungen (BENDa, NETsKy) offenbar nur fakultative Symptome darstellen.

Unser Kranker wies die hier aufgezählten Hauptsymptome auf. Auffällig war nur, daß zunächst eine muskuläre Hypotonie bestand, die sich erst nach einigen Jahren in eine allgemeine Rigidität verwandelte. Dafür mag wohl im wesentlichen der außerordentlich starke Befall des Kleinhirns verantwortlich zu machen sein, auf den auch die markante Ataxie zurückzuführen ist. Hierin zeigt unser Fall eine weitgehende Ähnlichkeit mit der Beobachtung von NeTsky. Zum erstenmal konnten wir wohl generalisierte Krampfanfälle bei Hallervorden-Spatzscher Krankheit feststellen, was bei den ausgedehnten herdförmigen Zerstörungen und diffusen Ganglienzellausfällen zu Schwierigkeiten in der Deutung der Pathogenese dieser Veränderungen führt. Es fragt sich nämlich, ob die laminären Ganglienzellausfälle, deren Genese auf Grund der histologischen Untersuchung nicht mehr erschlossen werden konnte, als Krampfschäden (ScHOLz) oder als Eigentümlichkeit der Grundkrankheit aufzufassen sind. Wir gehen wohl in der Annahme nicht fehl, wenn wir den umfangreichen Parenchymuntergang im wesentlichen auf die 
Grundkrankheit beziehen, da ja bereits vor dem Auftreten der Krampfanfälle ein schweres organisches Hirnsyndrom vorgelegen hatte.

Das pathologisch-anatomische Bild fanden wir in weitgehender Übereinstimmung mit den früheren Beobachtungen. Am markantesten und für die morphologische Diagnose bestimmend ist wohl die schon makroskopisch erkennbare Verfärbung von Pallidum und Zona rubra (= Zona reticulata) der Substantia nigra. Letztere war nur in den Fällen von Osman u. Schükrü und NetSky nicht mit dem bloßen Auge erkennbar, aber man kann sagen, daß eine histologisch erkennbare Pigmentanhäufung in diesen Gebieten zur Stellung der Diagnose unbedingt zu fordern ist. Wie andere Autoren, so sahen auch wir Pigmentansammlungen auch in anderen Stammgangliengebieten, speziell im oberen Teil des Claustrum. Ebenso wie Helfand u. Netsky, so fiel uns bei unserem Kranken die recht geringe Melaninbildung in der kompakten Zone der Substantia nigra auf, eine Tatsache, die Helfand mit veranlaßte, das Zustandsbild als ,status pigmentatus“ zu bezeichnen. Aber dieser Befund ist offenbar nicht pathognomonisch für die HALLERvordenSPatzsche Krankheit. Außerordentlich eindrucksvoll fanden wir den Untergang der Markfasern im Pallidum mit Wucherung der Astroglia, ein Befund, der aber nicht immer konstant ist.

Schon Hallervorden u. Spatz machten auf schwere diffuse Veränderungen in Groß- und Kleinhirnrinde aufmerksam, ein Befund, der nur von $\mathrm{Y}_{\text {AKOvLEV }}$ und von Fischer nicht erhoben wurde, bei unserem Kranken aber ganz besonders ausgesprochen war. Wir haben schon darauf hingewiesen, da $B$ in unserem Falle mit der Möglichkeit krampfgenetischer Parenchymausfälle zu rechnen ist. Die schweren Verödungen und Erbleichungen der Großhirnrinde glauben wir für den erheblichen Markfaserausfall in den großen Marklagern verantwortlich machen zu müssen. So schwere Markschäden, wie wir sie beobachtenkonnten, sind, soweit wir sehen, bisher noch nicht beschrieben worden.

Das Kleinhirn ist in der Literatur stiefmütterlich behandelt worden, obwohl es stets schwere Veränderungen aufzuweisen pflegt, welche einer sorgfältigen Bearbeitung wert sind. Die meisten Autoren begnügten sich mit der Bemerkung, da $\beta$ die Pukninje-Zellen reduziert sind, die granuläre Schicht gelichtet ist. Die Silberkarbonattechnik zeigt jedoch, daß es sich um eine Schädigung aller cerebellärer Systeme handelt, von denen einige nahezu völlig verödet sind. Diese Befunde sind jedoch so kompliziert, daß wir beabsichtigen, sie in einer weiteren Mitteilung zur Diskussion zu stellen. Befunde in der Medulla oblongata sind meist in Form von Nervenzelläsionen beschrieben worden. Diese haben auch wir nicht vermißt, darüber hinaus aber noch starke gliöse Reaktionen, wohl auf Grund sekundärer Markfaserdegenerationen, im Olivenflies und in der Substatia reticularis gesehen. 
Leider hat sich auch in unserem Fall kein Hinweis auf die Ätiologie der Krankheit ergeben. Selbst die Pathogenese der umfangreichen Veränderungen konnte nicht geklärt werden. Die darüber vorliegenden Auffassungen sind oftmals widersprechend. In diesem Zusammenhang möchten wir aber doch auf einen noch nicht erwähnten Befund verweisen, der uns möglicherweise einigen Aufschluß über die Pathogenese der geschilderten Veränderungen geben könnte. Wir waren aber bisher noch nicht in der Lage, diesen Tatsachen in so weitem Maße Rechnung zu tragen, daß sie hier schon näher ausgeführt werden könnten. Es handelt sich um eine Anhäufung von Pigmentstoffen in Ganglien- und Gliazellen aber auch im Ependym in den verschiedensten Regionen des Gehirns. Das Pigment ist im Plasma dieser Zellen in feinsten regelmäßigen Kügelchen gespeichert. Bei der NIssL-Färbung und in Silberschnitten konnten wir es nur mit Mühe erkennen, während es bei der Azur-Eosin-Färbung hervorragend dargestellt wird. Wir vermögen nicht zu sagen, ob es sich bei diesen Pigmentablagerungen um einen Zufallsbefund oder um eine bisher nicht als solche erkannte Eigenart der HaLLERvoRden-SPATzschen Krankheit handelt. Wir möchten aber diese Befunde vor allem in Hinblick auf die Beobachtungen Winkelmans (Retinitis pigmentosa) und HeLFANDs ,status pigmentatus" nicht unerwähnt lassen. Vielleicht werden sie doch noch einmal dazu beitragen können, die Pathogenese dieser eigenartigen und rätselhaften Krankheit zu klären.

\section{Zusammenfassung.}

Es wird über einen Jungen berichtet, der schon im frühen Kindesalter mit ataktischen und hyperkinetischen Störungen erkrankte. Die Sprache entwickelte sich niemals zu einem normalen Sprechen. Unter ständiger Zunahme dieser neurologischen Störungen, geistigem Verfall und späterem Auftreten von Rigidität und generalisierten Krampfanfällen führte die Krankheit im Alter von 14 Jahren zum Tode. Die anatomische Untersuchung ergab die typischen Befunde der HaLlervorden-Spatzschen Krankheit. Darüber hinaus wurde in Ganglien-, Glia- und Ependymzellen ein eigenartiges Pigment festgestellt, dem möglicherweise eine gewisse Bedeutung für die Pathogenese der beschriebenen Krankheit zukommen kann.

\section{Literatur.}

Benda, C. E.: Chronic Rheumatic Encephalitis, Torsion Dystonia and Hallervorden-SpatzDisease. Arch. Neur. Psych. 61, 137 (1949). - Eicke, W.: Neue Beobachtungen über die Hallervorden-Spatzsche Krankheit. Arch. Psychiatr. 111, 514 (1940). - Fischer, O.: Zur Frage der anatomischen Grundlage der Athetose double und der posthemiplegischen Bewegungsstörungen überhaupt. Z. Neur. 7, 463 (1911). - HaLlervorden, J.: Hallervorden-Spatzsche Krankheit. In Bunke: Handbuch der Geisteskrankheiten. 7. Teil. Berlin 1930. - Hallervorden, J., u. H. SPaTz: Eigenartige Erkrankung im extrapyramidalen System mit besonderer Beteiligung des Globus pallidus und der Substantia nigra. Ein Beitrag zu den Be- 
194 K. Scharenberg und R. DE Jong: Hallervorden-Spatzsche Krankheit.

ziehungen zwischen diesen beiden Zentren. Z. Neur. 79, 254 (1922). - HeLFand, M.: Status pigmentatus. Its Pathology and its Relation to Hallervorden-Spatz-Disease. J. Nerv. Ment. Dis. 81, 662 (1935). - Kaltnowsky, L.: Familiäre Erkrankung mit besonderer Beteiligung der Stammganglien. Mschr. Psych. 66, 168, (1927).

Netsky, M. G., D. Spiro and H. M. Zimmerman : Hallervorden-Spatz-Disease and Dystonia. J. Neuropath. 10, 125 (1951). — OsmaN, M., u. vaN SCHÜkrü: Beitrag zur Path. der Hallervorden-Spatzschen Krankheit. Z. Neur. 136, 178 (1935). Scholz, W.: Die Krampfschädigungen des Gehirns. Berlin 1951. - Winkelmann, N. W.: Progressive Pallidal Degeneration. A New clinicopathologic syndrome. Arch. Neur. Psych. 27, 1 (1932). - Yakovtev, P. I.: A Case of Myoclonus Epilepsy with Atrophy of Brain Stem and Hallervorden-Spatz type of Pathological Change $\mathrm{n}$ the Pallidum, Substantia nigra and Dentate Nucleus. Trans. Amer. Neur. Assoc. i942, 95.

Professor Dr. R. De Jong und Dr. K. Schakenberg, Ann Arbor/USA, University Hospital, The Neuropsychiatric Institute, University of Michigan. 